\section{P78 CONTEXTUAL SOCIAL DETERMINANTS OF MULTIMORBIDITY: A SYSTEMATIC REVIEW OF OBSERVATIONAL STUDIES}

${ }^{1} \mathrm{E}$ Ingram*, ${ }^{2} \mathrm{~S}$ Ledden, ${ }^{1} \mathrm{M}$ Gomes, ${ }^{3} \mathrm{~S}$ Hogarth, ${ }^{4} \mathrm{H}$ McDonald, ${ }^{2} \mathrm{D}$ Osborn, ${ }^{1} \mathrm{~J}$ Sheringham. ${ }^{1}$ Department of Applied Health Research, University College London, London, UK; ${ }^{2}$ Division of Psychiatry, University College London (UCL), London, UK; ${ }^{3}$ Public Health, London Boroughs of Camden and Islington, London, UK; ${ }^{4}$ London School of Hygiene and Tropical Medicine, London, UK

\subsection{6/jech-2020-SSMabstracts. 170}

Background Multimorbidity - the co-occurrence of multiple chronic conditions within the same individual - is a major global public health challenge.

People living in areas of greater deprivation have a higher burden of multimorbidity. It is not clear whether there are associations between other contextual (household and area-level) social determinants of health (SDoH), and multimorbidity. This study aims to systematically review the literature on associations between contextual SDoH and multimorbidity prevalence or incidence in the general population.

Methods A predefined search strategy (PROSPERO CRD42019135281) was run in 6 databases (MedLine, EMBASE, PsychINFO, Web of Science, CINAHL Plus and Scopus) in 2019. We included peer-reviewed studies published in English between 2010 and 2019, among general populations from high-income countries. We excluded studies if the SDoH did not align with the WHO's SDoH framework and studies conducted solely with institutionalised individuals or young people ( $<18$ years). A second reviewer independently screened all titles, abstracts and a subset of full-texts. Study quality was assessed using pre-specified criteria, and findings narratively synthesised.

Results From 3,298 records identified, 88 articles were reviewed on full-text and 41 papers met inclusion criteria (26 cross-sectional and 15 longitudinal). These spanned North America, Europe and Australasia. There was heterogeneity in definitions of multimorbidity; $34 / 41$ studies defined multimorbidity as $2+$ chronic conditions, whilst 8 used a cut-off point of $3+$ (and some operationalised both). 35/41 studies included physical and mental health conditions, 4 only physical conditions and 2 failed to include this information.

Household SDoH studies most commonly investigated associations with household income, with few on tenure, composition and other circumstances. Studies with area SDoH most commonly investigated measures of socioeconomic deprivation with fewer investigating rurality.

Studies consistently reported that individuals with the lowest household incomes and living in the most deprived areas had the highest prevalence or incidence of multimorbidity. For example, crude multimorbidity prevalence in the most and least deprived areas was $69.9 \%$ and $60.2 \%$ in one study reporting the highest figures, and $12.3 \%$ and $10.3 \%$ in another reporting the lowest. These associations varied according to the definition of multimorbidity. Amongst the few studies that investigated tenure, composition and rurality, findings were inconsistent. Possible reasons for mixed findings include data and methodological limitations.

Conclusion Current understanding of household SD of multimorbidity is limited. Application of a consistent definition of multimorbidity is needed for quantitative synthesis of findings. Better understanding of the social factors driving multimorbidity is needed to develop equitable services and effective prevention strategies.

\section{\begin{tabular}{l|l} 
P79 & TEN-YEARS RISK OF ALL-CAUSE MORTALITY IN THE
\end{tabular} ELSA-BRASIL COHORT: THE INTERSECTION OF RACE/ SKIN COLOR AND GENDER}

${ }^{1,2}$ SM Barreto*, ${ }^{1}$ FEG Oliveira, ${ }^{1,2} \mathrm{~L}$ Giatti, ${ }^{3} \mathrm{RH}$ Griep, ${ }^{1,2} \mathrm{LV}$ Camelo, ${ }^{4} \mathrm{D}$ Chor. ' $S o c i a l$ and Preventive Medicine, Universidade Federal de Minas Gerais, Belo Horizonte, Brazil; ${ }^{2}$ Post Graduate Program of Public Health, Universidade Federal de Minas Gerais, Belo Horizonte, Brazil; ${ }^{3}$ Laboratory of Health and Environment Education, Fundação Oswaldo Cruz, Rio de Janeiro, Brazil; ${ }^{4}$ National School of Public Health, Fundação Oswaldo Cruz, Rio de Janeiro, Brazil

\subsection{6/jech-2020-SSMabstracts. 17}

Background Historical, persistent and systematic disadvantages in health and socioeconomic indicators are observed for Brown and especially for Black individuals in Brazil when compared to White ones. Gender inequities are also present, being different for men and women, as men die more e more prematurely than women, while women generally present worse socioeconomic and morbidity markers than men. Analysis based on the intersectionality of race and gender have contribute to apprehend and deepened the understanding of the multiple and inseparable dimensions of the existing race and gender disparities in social and health factors. Although the number of studies using the intersection of race and gender are growing, few are longitudinal.

The main objective of this work was to investigate the role of race/skin colour and gender, as well as the intersection of these categories on the risk of death in the multicentric and multiracial Brazilian Longitudinal Study of Adults Health (ELSA-Brasil).

Methods A total of 14.365 civil servants from six Brazilian cities participants of the ELSA-Brasil cohort $(95.1 \%$ of original cohort) were followed up from study baseline (2008-2010) up to July/2018. Deaths were identified by annual interviews and death certificates. The associations between race/skin colour and gender separately, and between the intersection of race/ skin colour and gender on all-cause mortality were estimated using Cox proportional models. Adjustments for age (confounder) and socioeconomic, behavioural and health factors (potential mediators) at baseline were considered.

Results In total, 441 deaths (incidence: $3.1 \%$; 44/10.000 person-years) were identified. After age-adjustments, greater risks of death were observed for Black and Brown individuals in comparison with White ones (HR:1.95; 95\%IC:1.53-2.49 e HR:1.64; 95\%IC:1.32-2.03, respectively), and for men in relation to women (HR:1.96; 95\%IC:1.62-2.38). The intersectional analysis, considering White women as the reference category, Black women (HR:1.62; 95\%IC:1.13-2.41), White men (HR:1.71; 95\%IC:1.28-2.28), Brown men (HR:3.04; 95\%IC:2.24-4.12) and Black men (HR:3.74; 95\%IC:2.655.27) showed greater risk of death. After considering the adjustment for potential mediating factors, the magnitudes of the associations were attenuated, but the risk of death remained statistically greater for White men, Brown men and Black men, especially for the latter group.

Conclusion Race/skin colour and gender inequalities in the risk of death were confirmed and the extent of these inequities was found to be greater when social subgroups established in the intersectionality of race/skin color and gender are considered. 